\title{
Cuidadores: responsabilidades-obligaciones
}

\author{
Alma Elizabeth Ruiz Ríos,* Ma. Guadalupe Nava Galán**
}

\section{RESUMEN}

El objetivo de este ensayo es conocer las responsabilidades y obligaciones de los cuidadores desde diferentes disciplinas; esta reflexión busca influir en el cuidador para evitar complicaciones.

Palabras clave: Cuidador, responsabilidades, obligaciones, complicaciones.

\section{Caregivers: responsibilities-obligations}

\begin{abstract}
The objective of this paper know the responsibilities and obligations of caregivers from different disciplines; this reflection influences the caregiver to avoid complications.
\end{abstract}

Key words: Caregiver, responsibilities, obligations, complications.

$\mathrm{D}$ e acuerdo al diccionario de la Real Academia Española (RAE), responsabilidad hace referencia al compromiso u obligación de tipo moral que surge de la posible equivocación cometida por un individuo en un asunto específico. La responsabilidad es, también, la obligación de reparar un error y compensar los males ocasionados cuando la situación lo amerita. Es un valor que está en la conciencia de la persona, que le permite reflexionar, administrar, orientar y valorar las consecuencias de sus actos, siempre en el plano de lo moral. ${ }^{1}$

La persona responsable actúa conscientemente, siendo la causa directa o indirecta de un hecho ocurrido. Está obligada a responder por alguna cosa o alguna persona. También es quien cumple con sus obligaciones o pone cuidado y atención en lo que hace o decide. ${ }^{2}$
Así mismo, el Diccionario de la Real Academia Española señala que Cuidador/ra, es quien cuida a una persona, es una persona muy servicial, cuidadosa, pensativa y metida en sí. ${ }^{3}$ El cuidador principal es "aquel individuo que dedica la mayor parte del tiempo -medido en número de horas al díaal cuidado de dicho enfermo"3 (Dwyer, Lee y Jankowski, 1994). Un cuidador es la persona que se hace cargo, por cuenta propia o por encargo, de vigilar y atender a otra persona como un anciano, un bebé, un convaleciente, o una dependencia.

El cuidador, como lo entendemos en materia de dependencia, es la persona que facilita la vida y complementa la falta de autonomía de una persona dependiente, ayudándola en sus tareas diarias como aseo, alimentación, desplazamiento, entre otras. Al agotamiento físico y psíquico que produce

* Licenciada en Enfermería.

**Maestría en Enfermería en Educación.

Correspondencia: Alma Elizabeth Ruiz Ríos, Insurgentes Sur Núm. 3877, Col. La Fama, Deleg. Tlalpan, México, D.F., 14269, E-mail: rev.enf.neurol@gmail.com 
en los cuidadores la dedicación plena a una persona dependiente se le conoce como síndrome del cuidador.

\section{DESDE EL ENFOQUE ANTROPOLÓGICO}

Se ha definido al cuidador como "aquella persona que asiste o cuida a otra afectada de cualquier tipo de discapacidad, minusvalía o incapacidad que le dificulta o impide el desarrollo normal de sus actividades vitales o de sus relaciones sociales". ${ }^{5}$ Esta definición expresa la necesidad de tener en cuenta diferentes grados de responsabilidad en el cuidado de ancianos y la consecuente diferenciación entre cuidadores directos e indirectos. ${ }^{6}$

A partir de esta definición general de cuidadores, se impone la diferenciación de los cuidadores directos en formales e informales.

Los cuidadores "informales" no disponen de capacitación, no son remunerados por su tarea y tienen un elevado grado de compromiso hacia la tarea, caracterizada por el afecto y una atención sin límites de horarios. El apoyo informal es brindado principalmente por familiares, participando también amigos y vecinos (Flórez Lozano et al, 1997, Aguas, 1999). Algunos autores han señalado el carácter de cuidador principal (Anderson, 1987, en Flórez Lozano et al, 1997), por lo general desempeñado por el cónyuge o familiar femenino más próximo. Los cuidadores principales o primarios son los que asumen su total responsabilidad en la tarea, pasando por diferenciaciones progresivas a esta situación según la ayuda, formal o informal, que reciban. A diferencia de los cuidadores primarios, los secundarios no tienen la responsabilidad principal del cuidado de los ancianos (Stone et al, 1987, en Flórez Lozano et al, 1997). Más allá del interés semántico y descriptivo que representan estas diferenciaciones, interesa analizar el proceso valorativo implicado en las mismas y su incidencia en las prácticas que intervienen en la atención de los ancianos. La cooperación entre los diferentes tipos de cuidadores puede ser fundamental para la superación de la vulnerabilidad de ancianos y cuidadores familiares en el contexto hogareño, y adquiere especial relevancia en la institucionalización de larga estadía, cuando una intervención adecuada del equipo interdisciplinario puede potenciar la ayuda al anciano utilizando los recursos de la red familiar y social del mismo. En esta etapa surgen nuevas complejidades, ya que a la red informal que cuidaba del anciano se superpone un tipo de cuidador que la bibliografía considera como "formal" -me refiero al personal, profesionales y responsables del establecimiento geriátrico-, el que compartirá con el cuidador familiar el carácter directo y prolongado de la atención, aunque con límite de horarios y menor compromiso afectivo.
Función principal del cuidador: Permite que otras personas puedan desenvolverse en su vida diaria, ayudándolas a adaptarse a las limitaciones que su discapacidad funcional les impone. Son muchas las variables que se pudieran relacionar con el malestar del cuidador, pero está bien asentada la relación entre niveles altos de depresión y ser cuidador de un enfermo de Alzheimer. La falta de apoyo social y la falta de satisfacción con los lazos sociales se relacionan con un peor estado psicológico del cuidador.

Problemas psicológicos: En los diversos estudios que se han realizado, la salud mental de los familiares cuidadores primarios se ve más afectada que la salud física. Por ejemplo, en un estudio realizado por Rodríguez del Álamo y De Benito se observa ansiedad (nerviosismo, angustia, tensión y estrés), depresión o síntomas depresivos (tristeza, pesimismo, apatía), hipocondría y otras ideas obsesivas, ideación paranoide, angustia (pánico), ideas suicidas.

Problemas psicosomáticos: La gran mayoría de los cuidadores desarrolla problemas como dolores de cabeza y de otras zonas, falta de apetito, temblor fino, problemas gástricos, sensación de falta de aire o ahogo, arritmias y palpitaciones, sudoraciones y vértigos, alergias inmotivadas, trastornos del sueño (insomnio o sueño no reparador), fallas objetivas en la memoria (no sólo subjetivas), sobrecarga "estado psicológico que resulta de la combinación de trabajo físico), presión emocional, restricciones sociales, asî como las demandas económicas que surgen al cuidar dicho enfermo" (Dillehay y Sandys, 1990).

Burnout o síndrome del cuidador quemado: Este síndrome fue descrito primeramente en Estados Unidos en 1974. Consiste en un profundo desgaste emocional y físico que experimenta la persona que convive y cuida a un enfermo crónico incurable, tal como el enfermo de Alzheimer.

El cuidador que suele sufrirlo es aquel que llega a dedicarle casi todo su tiempo al paciente (incluso dejando de trabajar para cuidarle), actuando por lo general en forma solitaria (casi siempre hay otros familiares que suelen "lavarse las manos" y mantenerse en la periferia) y empleando estrategias pasivas e inadecuadas de resolución de problemas.

Se considera que se produce por el estrés permanente de tipo crónico (no el de tipo agudo de una situación puntual) en un batallar diario contra la enfermedad con tareas monótonas y repetitivas, con sensación de falta de control sobre el resultado final de esta labor, y que puede agotar las reservas psicofísicas del cuidador.

En base a estas circunstancias se desarrollan actitudes y sentimientos negativos hacia los enfermos a quienes se cuida: desmotivación, depresión-angustia, trastornos psicosomáticos, fatiga y agotamiento no ligado al esfuerzo, irritabilidad, despersonalización y deshumanización, comportamientos estereotipados con ineficiencia en resolver los 
problemas reales, agobio permanente con sentimiento de ser desbordado por la situación...

El cuidar es una situación que muchas personas a lo largo de sus vidas acaban experimentando. La experiencia de cada cuidador es única, ya que son demasiados los aspectos que hacen que esta experiencia difiera de cuidador a cuidador. El por qué se cuida y a quién se cuida, así como la relación previa con la persona cuidada, la causa y el grado de la dependencia del familiar, la ayuda que prestan otros miembros de la familia, las exigencias que se imponen los cuidadores son sin duda factores que intervienen en la calidad de vida tanto del enfermo como del cuidador.

Uribe (2006) refiere: "El cuidado diario y a largo plazo de un familiar enfermo, así se asuma de manera voluntaria y con cariño, conlleva riesgos para la salud de las personas que lo realizan, principalmente si toda la responsabilidad recae sobre una sola persona." 7 Por tanto, la gran función que desempeña el cuidador principal en estos pacientes puede ocasionar problemas de salud, no sólo de tipo físico sino también de tipo mental. Astudillo et al (2008) definen al cuidador primario como: "la persona que atiende en primera instancia las necesidades físicas y emocionales de un enfermo: papel que por lo general lo juegan el/la esposo/a, hijo/a, un familiar cercano o alguien que es significativo para el paciente". Su trabajo adquiere una gran relevancia para el grupo que atiende y rodea al enfermo conforme progresa la enfermedad, no sólo por la atención directa al paciente, sino también por su papel en la reorganización, mantenimiento y cohesión de la familia. ${ }^{8}$ Por su parte, Armstrong (2005) plantea: "El cuidador primario asume la responsabilidad total del paciente ayudándole a realizar todas las actividades que éste por sí mismo no puede llevar a cabo; en este rol es común que el cuidador sea un miembro de la red social inmediata (familiar, amigo o incluso vecino), y que, por lo general, no reciba ayuda económica ni capacitación previa para la atención del enfermo."9 Torres (2007), por su parte, sustenta: "La tarea de cuidar a un enfermo comprende a menudo la aparición de una amplia variedad de problemas de orden físico, psíquico y sociofamiliar, los cuales originan un auténtico síndrome que es necesario conocer y diagnosticar tempranamente para prevenir su agravamiento. En este proceso hay otros factores que pueden influir en la salud del cuidador; entre otros, habrán de considerarse su edad, género, parentesco, tiempo de dedicación y otras cargas familiares."10

La variedad e intensidad de la carga de los cuidadores se conoce cada vez con mayor detalle, sobre todo por la información que han arrojado abundantes encuestas. La literatura distingue la realización de actividades instrumentales de la vida cotidiana, tales como ayudar con el transporte, asear la casa, preparar las comidas, gestionar las finanzas, dar medicinas y la realización de actividades de la vida diaria tales como levantarlos de la cama o silla, vestirlos, llevarlos al aseo, ayudarles a comer y a manejar la incontinencia.

La intensidad del cuidado varía mucho: de ocho horas o menos a la semana, a todo el tiempo, día y noche. Los beneficios económicos sociales, psicológicos y terapéuticos del cuidado del familiar, en principio son obvios y se han documentado ampliamente en la literatura. ${ }^{11}$

El hacerse cargo de una persona dependiente durante largos periodos de tiempo puede desencadenar problemas de salud en el cuidador ya que, cuando está sobrecargado, comienza a notar alteraciones en todos los aspectos de su vida. Entre las alteraciones físicas se encuentran: cansancio, dolor de cabeza, dispepsia, vértigo, dificultades para dormir y dolores articulares.

De estos síntomas físicos, tiene particular relevancia la calidad del sueño que puede tener efectos nocivos sobre la salud. Chang y colaboradores (2007) realizaron un estudio donde concluyen que la calidad del sueño puede tener un impacto sobre varios aspectos de la calidad de vida de los cuidadores y que la ayuda al mejoramiento del sueño brinda capacidad para atender a los pacientes y a sí mismo. ${ }^{12}$

Dentro de las repercusiones en el terreno físico de los cuidadores primarios es conveniente retomar los resultados de un estudio realizado por Morales y colaboradores (2000) con 26 personas sometidas al juego de roles de esta naturaleza cuyo objetivo era analizar la repercusión que tiene la atención a enfermos crónicos y/o incapacitados sobre la salud de los cuidadores primarios; los investigadores encontraron que las principales patologías físicas que manifestaron los cuidadores fueron: cardiopatía en uno, cefalea en tres, cervicalgia en uno, mientras que 14 reconocieron lumbalgia como consecuencia de su tarea. Sólo uno no refirió patología alguna visiblemente manifiesta. En este trabajo se acepta el uso de antidepresivos, AINES. Asimismo, el 77\% presentó problemas para dormir. En estos casos, los somníferos eran consumidos por el $50 \% .^{13}$

\section{TIPOS DE CUIDADORES}

\section{Cuidador primario familiar}

\section{El esposo o la esposa como cuidador}

Cuando uno de los miembros de una pareja sufre un deterioro de salud y necesita ayuda para sus actividades de la vida diaria, el cuidador principal suele ser el miembro de la pareja con mejor salud.

Algunos de los rasgos comunes a estas situaciones son: cambio de roles, modificación de planes de futuro, revaluación de la relación interpersonal, sentimientos de ambivalencia y reajuste de la vida social. 
Como en cualquier situación de la vida que implique un cambio, la nueva situación que supone cuidar al marido o a la mujer puede resultar difícil y exige una constante adaptación. El hecho de que un miembro de la pareja sea el que tiene que dar ayuda y el otro el que la recibe puede crear tensiones en la pareja, al ser una relación menos recíproca. Es más fácil aceptar la ayuda del marido o de la mujer que la ayuda de familiares, amigos, vecinos o instituciones, en la medida en que se ve como una obligación transmitida de generación en generación, así como una muestra de cariño por los años de convivencia juntos. En el caso de que los cuidadores sean hermanos o hermanas de la persona dependiente también suele resultar más fácil aceptar su ayuda que la de otras personas.

Los maridos cuidadores reciben más ayuda de otros familiares y de las instituciones que las mujeres cuidadoras. En ocasiones, las mujeres cuidadoras se resisten a buscar o recibir ayuda de otros familiares, amigos, vecinos e incluso, de organismos oficiales. Estas mujeres, a menudo, acaban cuidando y sobrecargándose de trabajo más de lo que su salud y condiciones se lo permiten al no aceptar la ayuda de otros.

\section{Las hijas y los hijos como cuidadores}

Se encuentran a menudo con que deben atender también las necesidades de su familia (cónyuge e hijos), así como las propias.

En ellos se presentan los siguientes aspectos: fenómeno de inversión de roles, modificación de planes de futuro, revaluación de la relación interpersonal, sentimientos de ambivalencia, reajuste de la vida social y laboral.

Cuando la persona que cuida es la hija o el hijo, existe un vínculo natural familiar con la persona dependiente que "favorece" la disposición del cuidado. En la mayoría de las ocasiones, esto representa un fuerte impacto emocional para los hijos al darse cuenta de que el padre, la madre o ambos ya no pueden valerse por sí mismos, cuando eran personas independientes y el sostén del hogar. Este impacto también se ve afectado por el fenómeno de la inversión de roles. En cuanto el papel de cuidador que se asigna al hecho de ser padre o madre deja paso al papel de persona que necesita ser cuidada, y viceversa: el papel del hijo como alguien que es cuidado es sustituido por un papel de cuidador.

A los hijos les resulta muy difícil aceptar la situación de cuidar a sus padres, ya que normalmente implica algo imprevisto que les impide realizar actividades que pensaban llevar a cabo en un futuro inmediato.

Las hijas solteras, la hija favorita, la que tiene menos carga familiar o de trabajo, así como la hija que vive más cerca o la única mujer entre los hermanos, o el hijo favorito, suelen ser las personas sobre las que recae la responsabilidad del cuidado de sus padres. ${ }^{14}$

Normalmente, a medida que comienza a verse la necesidad de proporcionar cuidados al familiar dependiente, va perfilándose un cuidador principal que suele responder a las circunstancias de cada familia, sin necesidad de que se haya elegido por acuerdo explícito entre las personas que componen la familia. Cuando se asume el cuidado del familiar, muchas veces se piensa que va a ser una situación temporal, aunque, en muchas ocasiones, acaba siendo una situación que dura varios años y con una creciente demanda de cuidados. Gran parte de los hijos cuidadores no trabajan ni pueden pensar en buscar trabajo. Otros se ven obligados a reducir su jornada laboral. En los casos más extremos, se ven obligados a abandonar el trabajo por su situación de cuidador. Finalmente, los hijos e hijas de los cuidadores deben atender también a las necesidades de su familia (cónyuge e hijos), así como a sus propias necesidades. ${ }^{15}$

\section{Los padres como cuidadores}

Cuando es uno de los hijos quien por diversas circunstancias se convierte en una persona dependiente, el impacto emocional es mayor, presentándose regreso a un rol que ya se entendía superado; se caracteriza por modificación de planes de futuro, reevaluación de la relación interpersonal, sentimientos de ambivalencia y reajuste de la vida social. ${ }^{16}$

\section{FASES DE LA PERSONA AL VOLVERSE CUIDADOR}

Frecuentemente, en el inicio del cuidado la persona que cuida aún no es plenamente consciente de que es el miembro de la familia sobre el que va a recaer la mayor parte del esfuerzo y responsabilidades del cuidado; tampoco de que probablemente se encuentra en una situación que puede mantenerse durante muchos años y que, posiblemente, implique un progresivo aumento de dedicación en tiempo y energía. Poco a poco, sin darse cuenta, la persona va integrando su nuevo papel de cuidador en su vida diaria.

\section{FASES DE ADAPTACIÓN A LA SITUACIÓN DE CUIDADO $^{17}$}

\section{Fase 1: negación o falta de conciencia del problema}

En los primeros momentos del proceso de enfrentarse a la enfermedad crónica de una persona del entorno familiar es frecuente que se utilice la negación como un medio para controlar miedos y ansiedades. Así, es común encontrar que la persona se niega a aceptar las evidencias de que su familiar padece una enfermedad (o varias) que le lleva a necesitar la ayuda de otras personas para mantener su adaptación al 
medio. Otra forma de negar el problema es evitar hablar del deterioro o incapacidad del familiar enfermo.

Este estadio es, normalmente, temporal. Conforme el tiempo pasa y las dificultades de la persona enferma para mantener su autonomía funcional se hacen más evidentes, empieza a hacerse cada vez más difícil creer que se trata de una "enfermedad temporal". 18

\section{Fase 2. Búsqueda de la información}

En esta etapa, el cuidador comienza a buscar información sobre la enfermedad de su familiar, consecuencias, secuelas, medios de ayuda, segundas opiniones, medicina alternativa... Todo esto con la esperanza de que su familiar vuelva a ser el mismo.

En este momento, son muy comunes entre los cuidadores los sentimientos de "malestar" por la injusticia que supone el que les haya "tocado" a ellos vivir esa situación. El enfado o, en su versión más intensa, la ira, la culpa, son respuestas humanas completamente normales en situaciones de pérdida del control de la propia vida y sus circunstancias. Existen en la vida algunos hechos negativos que son inevitables y que no se pueden cambiar, y son situaciones de este tipo las que típicamente afrontan los cuidadores de personas dependientes. ${ }^{19}$

\section{Fase 3. Reorganización}

Conforme pasa el tiempo, los sentimientos de ira y enfado pueden continuar. La vida suele perder el sentido habitual hasta ese momento y las nuevas responsabilidades crean una carga pesada para la persona que cuida.

Sin embargo, algo de control se va ganando en esta etapa. Contando ya con la información y recursos externos de ayuda, con la voluntad de la familia para compartir la responsabilidad y con una idea más precisa de los problemas a los que hay que enfrentarse, la persona que cuida dispondrá de las herramientas necesarias para afrontar adecuadamente la situación del cuidado. Este periodo de reorganización tendrá como resultado el desarrollo de un patrón de vida más normal. La persona que proporciona los cuidados se sentirá progresivamente con más control sobre la situación y aceptará mejor estos cambios en su vida. ${ }^{20}$

\section{Fase 4. Resolución}

En esta fase aprenden a cuidar mejor de sí mismo; están más dispuestos a buscar la ayuda de otras personas con experiencias similares; suelen hacerse, en esta fase, más independientes, dedicando más tiempo a realizar actividades recreativas y sociales y pueden buscar y encontrar otras fuentes de apoyo emocional, tales como reforzar las amistades o crear nuevos amigos.

A pesar de que en esta fase las responsabilidades continúan aumentando en número e intensidad, si la persona que cuida logra una buena adaptación, podrá estar más serena que en los primeros momentos de la enfermedad. Se comienza a reconstruir una imagen de cómo era antes de que la enfermedad mostrase sus primeros signos, imagen que hará más confortable y significativa la labor de la persona que cuida. ${ }^{21}$

\section{COMPLICACIONES DEL CUIDADOR INFORMAL ${ }^{22}$}

La actitud del cuidador primario informal centrada en la atención a la persona con discapacidad o enferma puede dar lugar a una serie de implicaciones, entre las que se incluye el síndrome del cuidador, el cual se caracteriza por presentar problemas físicos, psicológicos y sociales; asimismo, complicaciones en el cuidado del paciente, de manera que resultan afectadas sus actividades de ocio, relaciones sociales, amistades, intimidad, libertad y equilibrio emocional, situación que da lugar a la denominada sobrecarga del cuidador.

La atención por parte del cuidador hacia el paciente representa un conjunto de emociones y conflictos, porque al cuidador suele resultarle inconcebible dejar al paciente en manos de otra persona; este sentimiento es originado por diversas emociones, como el amor, la culpa, el sentido del deber, el decoro y los valores religiosos. Normalmente, los cuidadores se sienten consternados si se ven forzados a renunciar a su papel, aunque sea por un periodo corto de tiempo.

A este respecto, diversos autores coinciden en plantear que la sobrecarga es un estado psicológico que resulta de la combinación de trabajo físico, presión emocional, las restricciones sociales, así como las demandas económicas que surgen al cuidar un enfermo crónico o con discapacidad. En algunas investigaciones se hace la diferencia entre sobrecarga objetiva y subjetiva, misma que se toma como base para el estudio. La carga subjetiva puede definirse como las reacciones emocionales ante la experiencia de cuidar. La carga objetiva se define como el grado de perturbaciones o cambios en diversos aspectos del ámbito doméstico y la vida de los cuidadores. Este último tipo de carga está relacionada con el desempeño de actividades del autocuidado de la vida diaria que el paciente no puede realizar por sí mismo (ejemplos: bañar, vestir, peinar, comer, entre otras), así como también con la práctica de actividades instrumentales (transportar, movilizar, llevar al médico, etcétera), mientras que la carga subjetiva está asociada con actitudes y reacciones emocionales que manifiesta el cuidador ante la experiencia de cuidar de una persona enferma.

Durante las últimas décadas ha tenido lugar una extensa investigación científica acerca de las implicaciones que tiene la carga del cuidado sobre los cuidadores informales de personas con alguna enfermedad crónica, degenerativa y dependiente. 


\section{CONSECUENCIAS DEL CUIDADO EN LA VIDA DEL CUIDADOR}

La vida de quienes atienden a una persona dependiente puede verse afectada de muchas maneras. Es frecuente que experimenten cambios en las siguientes áreas: en las relaciones familiares, en el trabajo y en su situación económica, en su tiempo libre, en su salud y en su estado de ánimo. También, aunque se considere que los cambios que ocurren durante el tiempo de cuidado son únicamente negativos, algunos también pueden ser positivos: la satisfacción de cuidar y cambios en las relaciones familiares.

\section{Cambios de las relaciones familiares}

Suelen aparecer conflictos por el desacuerdo entre la persona que cuida y otros familiares en relación con el comportamiento, decisiones y actitudes de unos y otros hacia la persona dependiente o por la forma en que se proporcionan los cuidados. Se presenta malestar con otros miembros de la familia debido a los sentimientos del cuidador principal acerca de que el resto de la familia no es capaz de apreciar el esfuerzo que realiza.

Otro cambio típico es la inversión de "papeles", ya que, por ejemplo, la hija se convierte en cuidadora de su madre variando así la dirección en la que se produce el cuidado habitual de padres e hijos. Este cambio de papeles requiere una nueva mentalidad respecto al tipo de relación que existía anteriormente entre padres e hijos y exige al cuidador, en definitiva, un esfuerzo de adaptación.

Es frecuente que la persona dependiente viva con el cuidador principal y su familia. Este cambio puede ser también una fuente de conflictos puesto que el resto de la familia también se ve afectada por la nueva situación, no siempre deseada. El cuidado de la persona dependiente implica muchas tareas, tiempo y dedicación. Un tiempo y una dedicación que en ocasiones la pareja o los hijos echan de menos para ellos. Para muchos cuidadores es conflictivo mantener un trabajo a la vez que se realizan las tareas de cuidado. En algunas ocasiones tienen la sensación de abandonar a la persona dependiente para ir al trabajo y en otras la de estar incumpliendo con el trabajo.

También son frecuentes las dificultades económicas, ya sea porque disminuyen los ingresos (disminución de la dedicación laboral) o porque aumentan los gastos derivados de los cuidados.

\section{Cambios en el tiempo libre}

Una parte sustancial del tiempo que antes se dedicaba al ocio, a los amigos, hay que dedicarla ahora a la tarea de cuidar. Es frecuente que el familiar cuidador perciba que no tiene tiempo para su ocio. Incluso es posible que no se dedique ese tiempo a sí mismo por los sentimientos de culpa que le produce pensar que si lo hace está abandonando su responsabilidad. La reducción de actividades en general y, sobre todo, de las actividades sociales es muy frecuente y está relacionada con sentimientos de tristeza y de aislamiento.

\section{Cambios en la salud}

Algo muy frecuente en los cuidadores es el cansancio físico y la sensación de que su salud ha empeorado desde que cuidan de su familiar. De hecho, no es una "sensación", sino que surge de comparar a personas que cuidan con personas sin esa responsabilidad: los cuidadores resultan con una peor salud.

Por otro lado, hay que tener en cuenta que la edad media de los cuidadores principales según la lectura es de 52 años y que un $20 \%$ son mayores de 65 años.

Por tanto, es muy probable que en ellos estén comenzando algunos de los cambios que conlleva el envejecimiento como disminución de la fuerza muscular, cambios en algunas estructuras que permiten el movimiento, etc.

Además, al estar sometidos a un esfuerzo físico mayor que cualquiera otra persona a esa edad, no es sorprendente que algunos de esos cambios se aceleren o que aparezcan precozmente.

\section{Cambios en el estado de ánimo}

La situación de cuidar a otra persona hace que muchos cuidadores experimenten sentimientos positivos. El simple hecho de que la persona a la que cuida y a la que quiere se encuentre bien puede hacerle experimentar esos sentimientos. La persona a la que se cuida puede mostrarle su agradecimiento y eso le hace sentir bien. Hay quien cree que ofrecer estos cuidados es una obligación moral y cumplir con ello lo hace sentirse satisfecho.

Cuidar a una persona dependiente puede ser, a pesar de las dificultades y la "dureza" de la situación, una experiencia muy satisfactoria para el cuidador.

\section{CONSECUENCIAS SOBRE EL ESTADO DE ÁNIMO ${ }^{23}$}

La experiencia de cuidar, día a día, a una persona dependiente, a menudo tiene consecuencias psicológicas negativas: sentimientos de tristeza, desesperación, indefensión y desesperanza, sentimientos de enfado e irritabilidad, de preocupación y ansiedad, y sentimientos de culpa. 


\section{LAS RELACIONES SOCIALES}

Las demandas del cuidado pueden hacer que los cuidadores vean reducido considerablemente su tiempo de ocio. Como consecuencia, es posible que las relaciones significativas con familiares y amigos disminuyan tanto en cantidad como en calidad. En el caso de que ya se haya producido la pérdida de los contactos sociales como consecuencia de las demandas de la situación de cuidado, sería recomendable que la persona que cuida buscase crear nuevos vínculos, nuevas relaciones positivas o amistades con quienes poder compartir experiencias positivas que potencien su bienestar y aligeren su carga.

\section{CONCLUSIONES}

El cuidador requiere de apoyo social, el cual debe ser proporcionado por la familia o las instituciones de salud, principalmente. De igual manera, requiere planificar su asistencia y cuidados al enfermo dejando un tiempo para sí mismo, estableciendo límites en el desempeño de su tarea, delegando funciones en otros miembros de la familia o en amigos, dejando de lado su papel de persona indispensable o de que nadie más podrá hacer las cosas mejor que ella, y sobre todo, recordando que eso no quiere decir que ame menos al enfermo.

El cuidador primario debe dejarse ayudar y procurar distribuir el trabajo en forma más equitativa y aceptar relevos para su descanso y pedir a los familiares, amigos o vecinos que le hayan manifestado alguna vez su deseo de apoyarle que le sustituyan para poder descansar o cambiar de actividad y tener tiempo para sí mismo, a fin de evitar el agotamiento emocional. Dar un paseo, distraerse, leer un libro, ir a comer con un amigo, hacerse un regalo, etcétera, son formas muy eficaces para combatir el estrés del cuidador. Tiene, sobre todo, que procurar seguir haciendo su vida normal.

Es saludable que mantenga su círculo de amigos y que pueda contar con una persona de confianza para hablar abiertamente sobre sus sentimientos y encontrar una salida a sus preocupaciones. También es una buena opción ponerse en contacto con otras personas que se encuentren en la misma situación para intercambiar impresiones.

En la misma línea de estos autores, se sugiere al cuidador primario un tiempo para sí mismo, donde pueda cambiar de actividad, como alternativa de manejo para combatir la carga que se pueda estar generando. De acuerdo a la literatura, la "terapia del arte" podría ser una opción a desarrollar durante este tiempo, a través de instrumentos artísticos, utilizando la expresión artística como recurso terapéutico, con la posibilidad de que la persona al pintar, esculpir, cantar, escribir, bailar, actuar... obtenga beneficios tales como la expresión de su problemática interna, la reducción de su tensión, el reconocimiento, manejo y control de sus emociones.
La terapia del arte, como herramienta psicoterapéutica, es relativamente nueva, y tiene grandes beneficios a nivel emocional, cognitivo y espiritual. El carácter paliativo de esta terapia radica en el hecho de que ante las peores circunstancias de la vida individual o social, el arte es la forma de no resignarse, de no dejarse en el abandono; más aún, es la forma de transformar creativamente lo más terrible de la realidad en formas bellas, en oportunidades de crecimiento y en circunstancias llenas de sentido.

\section{BIBLIOGRAFÍA}

1. González-Arnao V. Diccionario de la Real Academia Española. Edición Abreviada. Editorial Imprenta de Coson.

2. Larrañaga P. El concepto de responsabilidad. Editorial Fontamara, México, D.F., 2000 (fragmento de la tesis doctoral dirigida por el Prof. Manuel Atienza con el título El concepto de responsabilidad en la teoría del derecho contemporáneo, diciembre de 1996, Universidad de Alicante).

3. González-Arnao V. Diccionario de la Real Academia Española. Disponible en: http://lema.rae.es/drae/?val=cuidador

4. Dwyer L, Jankowski. Reciprocity elder satisfaction and caregivers stress and burden. Journal of Marriage and the Family. 56 (1): 35-43.

5. Flores LJA, Adeva CJ, García MC, Gómez MMP. Psicopatología de los cuidadores habituales de ancianos. Jano 1997; 3 (1218): 261-272.

6. De los Reyes MC. Construyendo el concepto cuidador de ancianos. IV Reunión de Antropología do Mercosul Foro de Investigación: Envejecimiento de la población en el Mercosur Noviembre. 2001. Curitiba. Brasil disponible en: http://www.redadultosmayores.com.ar/docsPDF/ Regiones/Mercosur/Brasil/06CuidadoresdeAncianos.pdf

7. Uribe ZP. Manual de apoyo para personas que brindan apoyo a otras personas. Secretaría de Salud, México 2006; 11.

8 Astudillo AW, Mendinueta AC. Necesidades de los cuidadores del paciente crónico. Guía de recursos sanitarios y sociales en la fase final de la vida en Gipuzkoa $7^{\text {a }}$ sección Madrid 235-255.

9. Amstrong P. Thinking it through: women, work and caring in the New Millennium. http://www.medicine. da.ca/mcewh 03-08-2005. In: Islas S, Ramos DR, Aguilar E, García G. Perfil psicosocial del cuidador primario informal del paciente con EPOC. Rev. Inst Nal Enf Resp Mex 2005; 19 (4): 266-271.

10. Torres F, Beltrán G, Martínez P, Saldivar G, Quesada C, Cruz T. Cuidar a un enfermo ¿pesa? Rev de Divulgación Científica de la Universidad Veracruzana. 2006; 19.

11. Cuidado Artesanal, la invención ante la adversidad, Carmen de la Cuesta Berjunmea, $1^{\text {a }}$ edición, Editorial Universidad de Antioquia. Colombia 2004; 162 .

12. Chang EW, Tsai YY, Chang TW, Tsao CJ. Quality of sleep and quality of life in caregivers of breast cancer patient. Psycho-Oncology.2007; 16 (10): 950-955.

13. Morales P, Muñoz R, Bravo V, Iniesta, Montero P, Olmos R. Problemas de salud de los cuidadores de enfermos incapacitados. Centro de Salud Totana Murcia 2000; 714-718.

14. Rojas M. Cuidar al que cuida. Claves para el bienestar del que cuida a un ser querido. Santillana Ediciones Generales, S.L. Madrid, 2006.

15. Rojas M, Ibidem.

16. Rojas M, Ibidem

17. Pérez MJ, Echauri M. Cuidar y cuidarse. Gobierno de Navarra, 2001.

18. Pérez MJ, Ibidem.

19. Pérez MJ, Ibidem.

20. Pérez MJ, Ibidem.

21. Torres F, Op Cit.

22. Torres F, Ibidem.

23. Cossio-Rodríguez I, Cotiello-Cueria Y, González-Sánchez M. Guía de atención a las personas que cuidan. Edita: Servicio de Salud del Principado de Asturias. España. 2006; 5-191. 